\title{
Wogitare
}

RELATO DE EXPERIÊNCIA

\section{CRIAÇÃO E APLICAÇÃO DE UMA ESTRATÉGIA GAMIFICADA NO ENSINO DE GRADUAÇÃO DE ENFERMAGEM}

\author{
Paulo Elias Gotardelo Audebert Delage ${ }^{1}$ (]) \\ Emanuelle Silva Mendes ${ }^{1} \oplus$ \\ Jully Greyce Freitas de Paula ${ }^{1}$ (๑) \\ lara Samily Balestero Mendes ${ }^{1} \oplus$ \\ Malena da Silva Almeida ${ }^{1} \odot$ \\ Fernanda de Nazaré Almeida Costa ${ }^{1}$ (i)
}

\section{RESUMO}

Objetivo: apresentar a construção, aplicação e avaliação de uma estratégia gamificada de ensino de metodologia científica a graduandos de enfermagem.

Método: diagnóstico dos problemas da disciplina Metodologia Científica do curso de enfermagem da Universidade do Estado do Pará; construção da estratégia; aplicação em seis turmas ao longo de três semestres, entre 2017 e 2018; avaliação dos impactos e aceitação.

Resultados: construção de um sistema de evolução heurístico, com impactos na assiduidade e pontualidade e autoavaliação positiva da estratégia.

Considerações Finais: a estratégia gamificada se mostrou eficaz na melhoria do engajamento de graduandos de enfermagem e com potencial para ser uma ferramenta pedagógica inovadora no ensino de enfermagem. Â adoção de uma estrutura de evolução heurística distancia esta estratégia de iniciativas artificiais com uso isolado de elementos de jogos. Avaliações mais rígidas, com grupo controle, ainda são necessárias.

DESCRITORES: Ensino; Educação em Enfermagem; Tecnologia Educacional; Metodologia; Jogos e Brinquedos.

\section{CREACIÓN Y APLICACIÓN DE UNA ESTRATEGIA DE GAMIFICACIÓN EN LA ENSEÑANZA DE GRADUACIÓN EN ENFERMERÍA}

\section{RESUMEN:}

Objetivo: presentar la construcción, aplicación y evaluación de una estrategia de gamificación para la enseñanza de metodología científica para estudiantes de graduación en enfermería. Método: diagnóstico de los problemas de la disciplina Metodología Científica del curso de enfermería de la Universidade do Estado do Pará; construcción de la estrategia; aplicación en seis clases a lo largo de tres semestres, entre 2017 y 2018; evaluación de los impactos y aceptación. Resultados: construcción de un sistema de evolución heurística, con impactos en asiduidad y puntualidad y autoevaluación positiva de la estrategia. Consideraciones Finales: la estrategia de gamificación demostró ser eficaz a la mejora del compromiso de los estudiantes de graduación en enfermería y con potencial para ser una herramienta pedagógica innovadora en la enseñanza de enfermería. La adopción de una estructura de evolución heurística distancia esta estrategia de iniciativas artificiales con el uso aislado de elementos de juegos. Se necesita asimismo evaluaciones más estrictas con grupo control.

DESCRIPTORES: Enseñanza; Educación en Enfermería; Tecnología Educacional; Metodología; Juego e Implementos de Juegos. 
Gamificação é o processo de fazer atividades no formato de jogos, proporcionando experiências típicas de jogo (1). Trata-se do planejamento de atividades adotando elementos de jogos, sem necessariamente construir ou usar jogos completos.

Apesar da multiplicidade de formatos de jogos existentes (digitais, tabuleiro, esportivos) e mecânicas (cooperativos, competitivos, individuais), quatro elementos são comuns a todos: (1) metas, que devem indicar um objetivo principal e intermediários; (2) regras, que determinem formas para se alcançar as metas, criando a noção de desafio; (3) feedbacks, que fornecem informações sobre progresso e desobediência às regras; e (4) a voluntariedade, caso contrário, seria um dever e não um jogo ${ }^{(2)}$.

Em sistemas gamificados, esses elementos têm contrapartes: (1) dinâmicas, que são a estrutura do sistema, incluindo metas, narrativas e senso de progressão; (2) mecânicas, que são as regras e a interatividade; e (3) componentes, que são os produtos do seguimento das mecânicas e incluem os pontos, troféus e prêmios ${ }^{(3)}$.

Estratégias gamificadas têm sido adotadas em áreas como corporativas ${ }^{(4)}$, saúde ${ }^{(5)} \mathrm{e}$ educação ${ }^{(6)}$, sendo particularmente profícuo neste último. Uma revisão demonstrou que, de 208 artigos sobre gamificação publicados entre 2008 e 2016, 94 eram aplicados ao ensino (45,19\%), em comparação, por exemplo, a 23 na saúde $(11,06 \%)$ e 17 no marketing $(8,17 \%)$. Apesar dessa profusão, há carência de trabalhos que avaliem seu sucesso, já que 97 artigos $(46,63 \%)$ apresentavam apenas argumentos conceituais para sua adoção( ${ }^{(7)}$.

Uma revisão sobre gamificação no ensino mostrou que, de 63 artigos sobre gamificação no ensino publicados entre 2014 e 2016, 44 (70\%) abordavam seu uso no ensino superior. Contudo, 20 dentre os $44(45,4 \%)$ relatavam iniciativas aplicadas a cursos de Tecnologia da Informação (TI) em contraste a cinco da saúde. Foi identificado que apenas $16(36,4 \%)$ gamificaram disciplinas inteiras e que todas essas eram online. Um único estudo usou dinâmicas e mecânicas de jogo, em contraste aos demais, que se limitaram a usar componentes desarticulados ${ }^{(3)}$.

A adoção de componentes isolados tem incorrido no modelo denominado PBL (do inglês Points, Badges and Leaderbords), onde são introduzidos elementos de jogos de maneira arbitrária e desarticulada, falhando em criar um sistema de jogo. Uma alternativa a esta tendência é a observação dos fatores motivacionais dos jogos, levando à criação de sistemas com múltiplos núcleos motivacionais ${ }^{(8)}$. Além disso, um dos fatores indicados como cruciais para o sucesso de estratégias gamificadas no ensino é a criação de sistemas de evolução heurística ${ }^{(3)}$, onde o progresso no "jogo" serve como condição e motivação para a continuidade do processo, simulando a sensação de evolução proporcionada pelos jogos eletrônicos, em detrimento da adoção de recompensas isoladas pelo cumprimento de tarefas específicas.

A adoção da gamificação no ensino superior tem crescido, mas ainda há pouca informação sobre seu real impacto e carência de iniciativas que a usem de maneira sistêmica e em disciplinas regulares. Assim, nosso objetivo é apresentar o processo de construção, aplicação e avaliaçãao de uma estratégia gamificada de ensino e seus impactos em seis turmas de graduação em enfermagem.

\section{MÉTODO}

O cenário foi o curso de graduação em enfermagem da UEPA (Universidade do 
Estado do Pará), na disciplina de Metodologia Científica, com carga horária semanal de três horas consecutivas. A escolha se deu por ser a disciplina ministrada pelo primeiro autor e por ser uma disciplina normalmente considerada enfadonha e monótona pelos alunos ${ }^{(9)}$.

A estratégia foi aplicada com seis turmas dos turnos matutino e vespertino, por três semestres entre 2017 e 2018. Todos os 131 discentes matriculados participaram da gamificação e foram convidados a integrar a pesquisa, sendo que 94 aceitaram o convite e assinaram o Termo de Consentimento Livre e Esclarecido (TCLE). Como o pesquisador principal também era professor da disciplina, o convite e coleta de dados foi apresentado por bolsistas e voluntários do grupo de pesquisa, garantindo assim o sigilo dos participantes e evitando qualquer tipo de coação ou constrangimento à participação na pesquisa.

A implementação se deu em três momentos: (1) diagnóstico da situação e criação; (2) aplicação; e (3) avaliação da estratégia. O diagnóstico foi pautado na percepção do professor de problemas observados anteriormente, com base nas avaliações informais realizadas no encerramento da disciplina em semestres pregressos, em que era realizada uma roda de conversa com os concluintes sobre sua percepção dos pontos positivos e negativos da disciplina; no levantamento do absenteísmo e percepção informal do professor da participação e engajamento dos discentes. Partindo deste levantamento, coube ao grupo de autores levantar elementos de jogo(2) relevantes para os problemas identificados e criar um sistema integrando esses elementos. A terceira etapa teve início no primeiro dia letivo de cada semestre, com a apresentação das regras, e era finalizada com o encerramento da disciplina e entrega da pontuação final ao término do período letivo. Após o encerramento de cada oferta da disciplina e entrega das notas, era aplicado aos discentes um questionário com uma escala do tipo Likert sobre o impacto da estratégia em seu desempenho.

O projeto foi aprovado no Comitê de Ética em Pesquisa da Faculdade de Enfermagem da UEPA, com o número 2.099.492.

\section{DISCUSSÃO}

\section{Construção e Aplicação}

Com base nas avaliações feitas pelos discentes em semestres anteriores, constatouse que havia excesso de aulas expositivas, o que, associado à natureza técnico-formal dos conteúdos, fazia com que as aulas fossem percebidas como cansativas e desinteressantes. Esta constatação pode ser corroborada por um absenteísmo alto, com uma média de 4,8 faltas por aluno nos três semestres anteriores. Outro problema era a falta de adoção dos materiais disponibilizados, incluindo os manuais e templates de trabalhos, com $58,3 \%$ dos alunos entregando trabalhos fora das normas da instituição. Também foi informalmente avaliada uma baixa pontualidade e saídas durante as aulas.

Para a construção da estratégia gamificada, foi adotada uma dinâmica de evolução heurística, onde as recompensas facilitavam o progresso no "jogo" e obtenção de novas recompensas; mecânicas de distribuição de pontos de experiência (XP-do inglểs Experience Points) por cumprimento de tarefas, feedbacks semanais e sistema de recompensas; e como componentes, prêmios por níveis e troféus.

A evolução heurística focava no desenvolvimento dos discentes, doravante chamados "jogadores", que acumulavam XP e avançavam de níveis, recebendo bônus que facilitavam seu desenvolvimento nas etapas posteriores. Trata-se de uma dinâmica comum em jogos de RPG, onde os jogadores destravam habilidades (boosters) à medida que evoluem ${ }^{(8)}$, e que vai além da simples distribuição de prêmios por cumprimento de tarefas, já que cria um senso de crescimento constante e as recompensas não são arbitrárias, mas se 
convertem em benefícios que auxiliam o avanço dentro do sistema. Assim, ao atingir o Nível 2, por exemplo, o jogador passaria a receber os templates dos trabalhos, aumentando as chances de receber mais pontos pelo seguimento das normas nas atividades futuras. Todos iniciavam o semestre no nível 0 e poderiam chegar até ao nível 10 (Figura 1):

\begin{tabular}{|c|c|c|}
\hline Nível & XP Necessário & Habilidade Desbloqueada \\
\hline 0 & 0 & Nenhuma \\
\hline 1 & 1.000 & Receber modelos de trabalhos (pdf) \\
\hline 2 & 2.500 & Receber modelos de trabalhos (doc) \\
\hline 3 & 4.500 & Acesso a documentos passados \\
\hline 4 & 7.000 & Correção antecipada dos trabalhos \\
\hline 5 & 10.000 & Desconto na palestra "Educação em Saúde" \\
\hline 6 & 14.000 & Ajuda de custo para os materiais da ação \\
\hline 7 & 19.000 & Negociação de prazos intermediários \\
\hline 8 & 27.000 & Correção dos slides da apresentação \\
\hline 9 & 35.000 & Conversão de XPs excedentes em nota \\
\hline 10 & 40.000 & Certificado: aluno(a) modelo $2017 / 2$ \\
\hline
\end{tabular}

Figura 1 - Exigência de XP para cada nível e suas recompensas. Belém, PA, Brasil, 2019 Fonte: Autores (2019)

Uma das mecânicas foi a distribuição de XP pelo cumprimento de tarefas(8). Ela é diretamente relacionada à evolução heurística, já que é por meio dela que os jogadores recebem os XP para sua evolução. Antes de cada tarefa era entregue uma súmula, indicando os valores em XP e os critérios para sua obtenção. Ao receber, por exemplo, instruções para elaboração de um relatório, eram apresentadas orientações sobre seu conteúdo, a pontuação prevista para cada seção e seus critérios (Figura 2).

\begin{tabular}{|c|c|c|}
\hline \multirow{5}{*}{$\begin{array}{l}\text { Introdução } \\
\text { (200 XP) }\end{array}$} & Apresentação clara do Tema e Objetivos & 30 \\
\hline & Contextualização do Trabalho & 30 \\
\hline & Fundamentação Teórica (geral e específica) & 80 \\
\hline & Citações condizentes com a ABNT & 50 \\
\hline & Fechamento da Introdução & 10 \\
\hline
\end{tabular}

Figura 2 - Operacionalização dos critérios de avaliação da introdução do relatório e seus valores em XP. Belém, PA, Brasil, 2019

Autores (2019) 
A outra mecânica adotada foi o sistema de feedbacks, que deve ser claro, sistemático e imediato(8). Infelizmente, dado o volume de atividades a serem analisadas, o feedback não era emitido imediatamente, sendo enviado para cada jogador todas as sextas-feiras por e-mail, com seu progresso em um arquivo de PowerPoint ${ }^{\circledR}$. Assim, o jogador dispunha de informações sobre seu nível; a quantidade de XP para o próximo nível; os XP distribuídos até o momento e na semana seguinte (Figura 3).

\begin{tabular}{|l|c|c|}
\hline ATIVIDADES + CRITÉRIOS & VALOR & PONTOS \\
\hline Elementos Obrigatórios & $\mathbf{8 0 0}$ & $\mathbf{6 0 0}$ \\
\hline Introdução & 200 & 200 \\
\hline Método & 200 & 100 \\
\hline Resultados & 200 & 100 \\
\hline Considerações Finais & 200 & 200 \\
\hline 1a Versão do Relatório & $\mathbf{5 0 0}$ & $\mathbf{4 2 5}$ \\
\hline Entrega no Prazo & 150 & 150 \\
\hline Formatação & 150 & 75 \\
\hline Seções Obrigatórias & 200 & 200 \\
\hline \multicolumn{1}{|c|}{ TOTAL } & $\mathbf{1 , 3 0 0}$ & $\mathbf{1 , 0 2 5}$ \\
\hline
\end{tabular}

\begin{tabular}{|l|c|c|}
\hline PONTOS DE EXPERIÊNCIA (XP) & SEMANA 3 & TOTAL \\
\hline Disponibilizados & 1,300 & 5,800 \\
\hline Conquistados & 1,025 & 5,525 \\
\hline
\end{tabular}

\begin{tabular}{|l|c|}
\hline \multicolumn{2}{|c|}{ NÍVEIS } \\
\hline Nível Atual & 3 \\
\hline Nível máximo possível & 3 \\
\hline XP para próximo nível & 1,475 \\
\hline
\end{tabular}

\begin{tabular}{|l|c|}
\hline \multicolumn{2}{|c|}{ PRÓXIMA SEMANA } \\
\hline XP Disponíveis & 2,300 \\
\hline Nível máximo possível & 4 \\
\hline
\end{tabular}

Figura 3 - Exemplo de distribuição de pontos de experiência e progresso semanal de um jogador na terceira semana letiva. Belém, PA, Brasil, 2019

Autores (2019)

Os componentes eram prêmios e troféus, ambos apresentados no mesmo arquivo. Os prêmios eram representados por imagens de jogos clássicos e uma indicação do seu efeito. Os troféus eram apresentados em formato de lista a ser completada.

Os prêmios foram baseados em práticas já adotadas em versões anteriores da disciplina ofertada em semestres anteriores, como, por exemplo, a disponibilização de templates. A diferença é que anteriormente esses templates eram entregues no início do semestre e indicados como material obrigatório, ao passo que na gamificação eles eram conquistados e vistos como benefícios pelo progresso. A mudança na forma de apresentação e função deste tipo de material se baseou no princípio da escassez, um dos núcleos motivacionais da gamificação(8).

Os troféus são marcadores de conquistas. Seu apelo está relacionado ao colecionismo(8). No início do semestre, era entregue uma lista dos troféus a serem conquistados, que eram "desbloqueados" à medida que alguns critérios eram satisfeitos. Existiam nove troféus de bronze (fáceis); seis de prata (intermediários); cinco de ouro (difíceis); e um de platina, que dependia da conquista de todos os troféus.

\section{Avaliação da Estratégia Gamificada}


Muito do conteúdo expositivo foi substituído por atividades às quais eram atribuídos os XP. A aula sobre referências, por exemplo, assumiu um formato de gincana e se tornou a aula mais elogiada pelos discentes, reforçando os impactos positivos de metodologias ativas de ensino(10).

O absenteísmo caiu de 4,8 para 1,1 falta durante o período da gamificação e foi observada melhoria na pontualidade e redução de saídas de sala, reforçando o papel de aumento do engajamento, esperado da gamificação( ${ }^{(3)}$.

A proporção de discentes que ignoravam os templates caiu de $58 \%$ para $30 \%$. Essa mudança pode se dever tanto ao valor agregado às atividades, que além de nota passaram a valer XP, quanto à mudança de função do template. Tradicionalmente os templates eram apenas uma exigência acadêmica e agora passaram a ser uma conquista, gerando um efeito de impulso ${ }^{(8)}$.

A avaliação da estratégia gamificada foi completada por 94 discentes $(71,8 \%$ do total de concluintes). 84 (89\%) consideraram que a estratégia afetou positivamente seu desempenho; $83(88,3 \%)$ que afetou sua assiduidade; $81(86,2 \%)$ que afetou sua pontualidade; 87 (92,5\%) que teve impacto positivo na participação nas aulas; e $89(94,7 \%)$ que ela afetou seu capricho. A avaliação dos elementos mostrou que $79(84 \%)$ consideraram que os prêmios tiveram impacto no seu desempenho; 78 (83\%) consideraram os troféus como positivos; e $83(88,3 \%)$ avaliaram como alto o impacto dos feedbacks semanais. Esses dados corroboram os achados em pesquisas que usaram metodologias similares ${ }^{(3)}$.

\section{CONSIDERAÇÕES FINAIS}

A adoção da gamificação no ensino superior tem aumentado, mas iniciativas de gamificar disciplinas inteiras, adotando dinâmicas e mecânicas em detrimento de elementos isolados, ainda são $\operatorname{raras}^{(3)}$. Esta experiência demonstrou que partir de um diagnóstico e criar um sistema individualizado produziu resultados positivos.

A dinâmica de evolução heurística gerou um senso de crescimento, de modo que os $\mathrm{XP}$ assumiram uma função distinta das notas. Enquanto essas têm um caráter pregresso e estanque, aqueles são uma oportunidade de crescimento e evolução.

A autoavaliação do impacto da estratégia também mostrou um resultado positivo. Considerando se tratar de uma disciplina comumente preterida pelos discentes, a adoção da estratégia foi útil não apenas por produzir maior engajamento, mas por melhorar a percepção dos discentes sobre a disciplina.

Iniciativas como esta são úteis ao demonstrar o impacto positivo da substituição de estratégias aversivas e coercitivas por estratégias pautadas no senso de autorrealização e progresso pessoal, na criação de ambientes favoráveis à aprendizagem. Isto pode ser particularmente útil em disciplinas transversais do início do curso, quando não há uma clareza da importância desses conteúdos, o que pode levar a uma desmotivação pelos alunos.

Apesar dos resultados iniciais positivos e percepção favorável dos discentes, ainda é necessário realizar novas aplicações, com avaliações mais rígidas do impacto no empenho e desempenho acadêmico, preferencialmente envolvendo procedimentos quaseexperimentais com grupos controle. 
Agradecemos à Fundação Amazônia de Amparo a Estudos e Pesquisas (FAPESPA - Edital 024/2017) e à Coordenação de Aperfeiçoamento de Pessoal de Nível Superior (CAPES - Processo 1628033) pela concessão de Bolsa de Iniciação Científica e Mestrado à segunda e terceira autoras, respectivamente.

\section{REFERÊNCIAS}

1. Werbach K. (Re)Defining gamification: a process approach. In: Spagnolli A, Chittaro L, Gamberini, L. Persuasive technology [Internet]. Padua, Italy: Springer International Publishing; 2014. [acesso em 05 mai 2017]; Disponível em: http://link.springer.com/chapter/10.1007/978-3-319-07127-5 23.

2. Mcgonigal, J. A realidade em jogo. Rio de Janeiro: Best Seller; 2012.

3. Dichev C, Dicheva D. Gamifying education: what is known, what is believed and what remains uncertain: a critical review. Int. J. Educ. Technol. High. Educ [Internet]. 2017 [acesso em 13 nov 2019]; 14(9). Disponível em: http://doi.org/10.1186/s41239-017-0042-5.

4. Celano J, Leite W, Silva FP da. A gamificação e a necessidade constante de mudanças nas organizações. Comunicação e mercado [Internet]. 2015 [acesso em 13 nov 2019]; 4(10). Disponível em: https://www.unigran.br/dourados/mercado/paginas/arquivos/edicoes/10/1.pdf.

5. Johnson D, Deterding S, Kuhn KA, Staneva A, Stoyanov S, Hides L. Gamification for health and wellbeing: a systematic review of the literature. Internet Interventions [Internet]. 2016 [acesso em 13 nov 2019]; 6. Disponível em: https://doi.org/10.1016/j.invent.2016.10.002.

6. Klock ACT, Carvalho MF de, Rosa BE, Gasparini I. Análise das técnicas de gamificação em ambientes virtuais de aprendizagem. RENOTE [Internet]. 2014 [acesso em 13 nov 2019]; 12(2). Disponível em: https://seer.ufrgs.br/renote/article/view/53496/33013.

7. Bozkurt A, Durak G. A systematic review of research: in pursuit of homo ludens. Int J Game Base Learn. [Internet]. 2018 [acesso em 13 nov 2019]; 8(3). Disponível em: http://doi.org/10.4018/ijgbl.2018070102.

8. Chou YK. Actionable gamification: beyond points, badges and leaderboards. Los Angeles: Octalysis Media; 2015.

9. Rodrigues FWA, Ramos ABB. Metodologia científica: análise e reflexão sobre a percepção dos graduandos. Int. J. Educ. Teach [Internet]. 2019 [acesso em 20 maio 2020]; 2(1). Disponível em: http://doi. org/10.31692/2595-2498.v2i1.90.

10. Diesel A, Baldez ALS, Martins SN. Os princípios das metodologias ativas de ensino: uma abordagem teórica. Revista Thema [Internet]. 2017 [acesso em 18 maio 2019]; 14(1). Disponível em: http://periodicos. ifsul.edu.br/index.php/thema/article/view/404. 


\section{COMO REFERENCIAR ESTE ARTIGO:}

Delage PEGA, Mendes ES, Paula JGF de, Mendes ISB, Almeida M da S, Almeida F de N. Criação e aplicação de uma estratégia gamificada no ensino de graduação de enfermagem. Cogitare enferm. [Internet]. 2021 [acesso em "colocar data de acesso, dia, mês abreviado e ano"]; 26. Disponível em: http://dx.doi.org/10.5380/ ce.v26i0.70221.

Recebido em: 18/11/2019

Aprovado em: 23/07/2020

Editora associada: Susanne Elero Betiolli

\section{Autor Correspondente:}

Paulo Elias Gotardelo Audebert Delage

Universidade do Estado do Pará - Belém, PA, Brasil

E-mail: gotardelo@gmail.com

\section{Contribuição dos autores:}

Contribuições substanciais para a concepção ou desenho do estudo; ou a aquisição, análise ou interpretação de dados do estudo - PEGAD, ESM, JGFP

Elaboração e revisão crítica do conteúdo intelectual do estudo - PEGAD, JGFP, FNAC

Aprovação da versão final do estudo a ser publicado - ESM, FNAC

Copyright () 2021 Este é um artigo em acesso aberto distribuído nos termos da Licença Creative Commons Atribuição, que permite o uso irrestrito, a distribuição e reprodução em qualquer meio desde que o artigo original seja devidamente citado. 